\title{
Moderne Laserzahnheilkunde gefördert
}

Die Entwicklung des SIROLaser Blue mit kurzer Wellenlänge verbessert die Einsatzmöglichkeiten des Lasers in der Zahnheilkunde. Um der Verwendung der vorteilhaften LaserTechnologie zum Durchbruch zu verhelfen, unterstützte Sirona mit den „Sirona Laser Days“ vom 8. bis 10 . Oktober im oberitalienischen Brescia das Kennenlernen und den Austausch der internationalen Community in der Laserzahnheilkunde.

Die neue blaue Lasertechnologie verbessert noch einmal die Leistung, vor allem in der minimalinvasiven Chirurgie, etwa bei der Freilegung eines Implantats. Das war die zentrale Botschaft der 2. Laser Days. Ein zentraler Schwerpunkt auf der Tagung waren die neuesten wissenschaftlichen Erkenntnisse zur blauen Lasertechnologie. Der SIROLaser Blue überzeugt vor allem in der Chirurgie durch seine gute
Schneidleistung. Der blaue Laser wird um ein Vielfaches besser im Gewebe absorbiert als der infrarote. Bei chirurgischen Eingriffen dringt blaues Laserlicht wegen seiner kürzeren Wellenlänge nicht so tief in das Gewebe ein und wird dort weniger gestreut. Während das blaue Laserlicht wegen der verbesserten Absorption vor allem in der Weichgewebschirurgie eingesetzt wird, deckt der SIROLaser Blue mit 2 weiteren Wellenlängen das vollständige Behandlungsspektrum von Diodenlasern ab. So lässt er sich mit infrarotem Laserlicht in einer Wellenlänge von 970nm für die klassischen, keimreduzierenden Indikationen in der Endodontie und Parodontologie einsetzen. Für eine Reihe weiterer Anwendungen der modernen Laserzahnheilkunde eignet sich die 3. Wellenlänge. Mit einer Wellenlänge von 660nm und einer niedrigen Leistung von bis zu 100 Milliwatt ermöglicht er die Low-Level-Lasertherapie zur Desensibilisierung, Beschleunigung der Wundheilung und zur Bekämpfung von Entzündungen.

Nach einer Pressemitteilung der Sirona Dental GmbH , A - Wals bei Salzburg www.sirona.com 